\title{
Bio-Efficacy of Phyto-Pesticides against Major Insect- Pest of Organically Grown Tomato
}

\author{
Ajay Deep Singh, P. Prathyusha*, Y. P. Malik and R. K. Dwivedi \\ Department of Entomology, Chandra Shekhar Azad University of Agriculture and \\ Technology, Kanpur-208002, Uttar Pradesh, India \\ *Corresponding author
}

\section{A B S T R A C T}

The experiment was carried out in rabi season 2018-19 in the Insectary of Department of Entomology, Chandra Shekhar Azad University of

Keywords

Bio-efficacy, Phytopesticides, Tomato, Helicoverpa armigera

Article Info

\section{Accepted:}

16 April 2021

Available Online:

10 May 2021
Agriculture and Technology, Kanpur. On the basis of present investigation, it is concluded that the different treatments Spinosad 45 SC $(0.015 \%)$, neem and bakain were most effective treatments against the major insect pests of tomato variety Type- 6 . Spinosad $45 \mathrm{SC}(0.015 \%)$ was found most effective followed by neem and bakain gave $90.46 \%, 87.06 \%$ and $84.00 \%$ healthy tomato fruits, respectively and $9.53 \%, 12.10 \%$ and $16.0 \%$ tomato fruit infestation on number basis, respectively considered the most effective treatments against $H$. armigera. neem and bakain were most effective treatments against aphids, A. gossypii and thrips, Scirtothrips dorsalis and spinosad 45 SC $(0.015 \%)$ for fruit borer, $H$. armigera. Spinosad 45 SC $(0.015 \%)$ provided highest fruit yield with $251.10 \mathrm{q} /$ ha followed by neem and bakain 241.11 and $225.55 \mathrm{q} / \mathrm{ha}$, respectively.

\section{Introduction}

Tomato (Lycopersicon esculentum Mill.) is considered significant vegetable crop in the world after potato (Marasini and Paudel, 2017), but the high acidic content of the tomato makes it a prime candidate for canning and ranks first in the list of canned vegetables and known as poor man's apple (Mehta, 2017). It is popularly used for both commercial and home use purposes. The processed products like tomato paste, tomato juice, tomato catchup and whole peel-tomato are produced in the country for local market and export.

In India, tomato occupies an area of 808.5 thousand hectare with production of 19696.9 thousand metric tonnes and productivity of 24.4 metric tonnes per hectare (Horticulture Statistics Division, Department of Agriculture, Co-operation \& Farmer's Welfare, 2016-17). Major tomato growing states in India are 
Bihar, Karnataka, Orissa, Maharashtra and Andhra Pradesh. In Uttar Pradesh, it occupies an area of 20.88 thousand hectare with production of 826.32 thousand metric tonnes and productivity of 39.57 metric tonnes per hectare (Horticulture Statistics Division, Department of Agriculture, Co-operation \& Farmer's Welfare, 2016-17).

Tomato crop is being damaged by a total of 41 insect-pests species belonging to 21 families which included the defoliators (Spodoptera litura, Monolepta andrawesi, Poekilocerus pictus and Atractomorpha crenulata), leaf miner (Liriomyza trifolii), sucking insect-pests (Bemisia tabaci, Aphis gossypii, Myzus persicae and Nezara viridula), stem feeders, Euzophera perticella and Leucinodes orbonalis and fruit borers, Helicoverpa armigera and Othreis fullonica (Eudocima fullonica) (Reddy and Kumar, 2004). The sucking pests white flies, thrips and aphids not only feed on foliage, stem and fruits in deteriorating the quality, but also act as the vector for disseminating tomato virus. These pests infest and hamper growth of the fruits. The yield losses in tomato crop due sucking pests aphid (Aphis gossypii Glover) is $20-40 \%$ (Sutton, 1991) and thrips (Scirtothrips dorsalis Hood) is more than 90\% (Kumar N.K., 1995). The tomato fruit borer (Helicoverpa armigera Hubner) is a key pest as it attacks the cashable part of the plant i.e. fruits, Yield losses in tomato due to tomato fruit borer is estimated around 22 to $38 \%$ in India (Dhandapani et al., 2003).

Pesticides produced from natural products have been recently attracting the attention of many scientists to avoid the problems caused by synthetic compounds. They are highly interested in their chemical constituents and biological properties (Abou-Yousef et al., 2010). Organic production of perishable foods seems to be the best alternative with least health hazards in keeping harmony with the nature. Many indigenous plant extracts (phytopesticides) have been tried and recommended for insect-pests management in different field crops (Vyas et al., 1999; Gurjar et al., 2012; Kumar et al., 2012; Shah et al., 2013), which are safer to environment, natural enemies, humans and other animals along with low to moderate mammalian toxicity (Hassan, 1992).

\section{Materials and Methods}

The crop was raised organically without application of synthetic fertilizers as well as plant protection measures. Application of FYM and neem cake was done at the time of field preaparation. Foliar spraying of Panchagavya (3\%) at 15, 45 and 75 days after transplanting (DAT) as well as soil drenching of Panchagavya (50 1/ha with irrigation) of Panchgavya was done at 30,60, and 90 DAT in lieu of synthetic fertilizers to raise a good crop as per Gore and Sreenivasa (2011). Weed free crop was raised and irrigation was done as per requirement of the crop to achieve good yield.

\section{Preparation of Panchgavya}

Panchagavya is an organic product recommended for crop improvement in organic agriculture (Sangeetha and Thevanathan, 2010). Panchgavya means "mixture of five products (cow dung, cow urine, milk, curd and ghee) of cow". Of these the three direct constituents are cow dung, cow urine and milk and two derived products are ghee and curd. Panchgavya was prepared by mixing cow dung (5 kg), cow urine (3 litres), cow milk (2 litres), curd made from cow milk (2l), ghee made from cow milk (1 $\mathrm{kg}$ ), sugarcane juice (3 litres), tender coconut water (3 litres) and ripened banana (12 Nos.), Prabhu (2009). In a wide mouthed mud vessel $5 \mathrm{~kg}$ of Cow dung and $500 \mathrm{~g}$ of Cow ghee were mixed thoroughly and kept it for 3 days. After 3 days, the remaining ingredients were 
added and kept it for 19 days with regular mixing both in morning and evening hours daily. The contents were stirred twice a day for about 20 minutes both in the morning and evening to facilitate aerobic microbial activity. On the $20^{\text {th }}$ day 20 liters Panchagavya is ready for use.

\section{Preparation of phyto-pesticides}

$100 \mathrm{~g}$ leaf of each test plant were ground with $100 \mathrm{ml}$ of distilled water with the help of mixer and a fine paste was prepared, which was left for three days for fermentation. The paste was filtered through a four layered muslin cloth to obtain a concentration for the spraying, which was termed as phyto-pesticide of $100 \%$ purity. This concoction was sprayed @ 5\% (50 ml/1 of water) twice at fortnightly interval for the management of different insect-pests. Detailed of description of different plants used are being given in Table1 .

Data on the population of aphid and thrips were taken on 5 randomly selected plants from each plot treated with phyto-pesticides, separately along with control plot. Average intensity of aphids and thrips/plant and fruit borer (larvae/plant) was recorded.

\section{Results and Discussion}

Different treatments were applied at fortnight interval starting from $15^{\text {th }}$ January and data were recorded on aphid, thrips and fruit borer and the results was portrait Table-2, Table-3 and Table-4, respectively.

\section{Aphid (Aphis gossypii Glover)}

Data depicted in table 2 on aphid intensity revealed that the overall mean population was calculated considering the average of all observations which varied between 36.90 to 101.79 aphids/plant. Neem leaf concoction treated tomato crop received significantly lowest aphid intensity 36.90 aphids/plant which was on par with 43.04, 44.66 and 46.27 aphids/plant recorded on madar, castor and lantana leaves decoction sprayed crop respectively.

Table.1 Details of plants used for preparation of phyto-pesticides

\begin{tabular}{|c|c|c|c|c|}
\hline $\begin{array}{c}\text { Common } \\
\text { name }\end{array}$ & Scientific name & Order & Family & $\begin{array}{c}\text { Plant part } \\
\text { used }\end{array}$ \\
\hline Castor & Ricinus communis & Malpighiales & Euphorbiaceae & Leaves \\
\hline Madar & Calotropis gigantea & Genitianales & Asclepiadaceae & Leaves \\
\hline Safeda & Eucalyptus globulus & Myrtales & Myrtaceae & Leaves \\
\hline Lantana & Lanatana camara & Lamiales & Verbenaceae & Leaves \\
\hline Drumstick & Moringa oliefera & Brassicales & Moringaceae & Leaves \\
\hline Neem & Azadirechta indica & Sapindales & Meliaceae & Leaves \\
\hline Congress grass & $\begin{array}{c}\text { Parthenium } \\
\text { hysterophorus }\end{array}$ & Asterales & Asteraceae & Leaves \\
\hline Bakain & Melia azadirach & Sapindales & Meliaceae & Leaves \\
\hline Datura & Datura stramonium & Solanales & Solanaceae & Leaves \\
\hline Kaner & Nerium oleander & Gentinales & Apocynaceae & Leaves \\
\hline
\end{tabular}


Table.2 Efficacy of phyto-pesticides against aphid infestation on tomato during rabi , 2018-19

\begin{tabular}{|c|c|c|c|c|c|c|c|}
\hline \multirow{3}{*}{$\begin{array}{l}\text { Treat. } \\
\text { Symbol }\end{array}$} & \multirow[t]{3}{*}{ Treatments } & \multicolumn{5}{|c|}{ Aphid/plant } & \multirow{3}{*}{$\begin{array}{l}\text { Overall mean } \\
\text { of two sprays }\end{array}$} \\
\hline & & & \multicolumn{2}{|c|}{$\mathbf{1}^{\text {st }}$ spray } & \multicolumn{2}{|c|}{$2^{\text {nd }}$ spray } & \\
\hline & & $1 \mathrm{DBS}$ & 7 DAS & 14 DAS & 7 DAS & 14 DAS & \\
\hline $\mathrm{T}_{1}$ & Castor & $\begin{array}{c}61.33 \\
(7.86)^{*}\end{array}$ & $\begin{array}{l}47.74 \\
(6.95)\end{array}$ & $\begin{array}{l}52.81 \\
(7.30)\end{array}$ & $\begin{array}{l}43.81 \\
(6.66)\end{array}$ & $\begin{array}{l}34.31 \\
(5.90)\end{array}$ & $\begin{array}{l}44.66 \\
(6.72)\end{array}$ \\
\hline $\mathrm{T}_{2}$ & Neem & $\begin{array}{l}65.66 \\
(8.13)\end{array}$ & $\begin{array}{l}40.49 \\
(6.40)\end{array}$ & $\begin{array}{l}45.28 \\
(6.77)\end{array}$ & $\begin{array}{l}35.68 \\
(6.01)\end{array}$ & $\begin{array}{l}26.18 \\
(5.17)\end{array}$ & $\begin{array}{l}36.90 \\
(6.12) \\
\end{array}$ \\
\hline $\mathrm{T}_{3}$ & safeda & $\begin{array}{l}69.00 \\
(8.34)\end{array}$ & $\begin{array}{l}58.63 \\
(7.69)\end{array}$ & $\begin{array}{l}63.57 \\
(8.00)\end{array}$ & $\begin{array}{l}53.97 \\
(7.38)\end{array}$ & $\begin{array}{l}44.47 \\
(6.71)\end{array}$ & $\begin{array}{l}55.16 \\
(7.46)\end{array}$ \\
\hline $\mathrm{T}_{4}$ & Kaner & $\begin{array}{l}64.00 \\
(8.03)\end{array}$ & $\begin{array}{l}60.73 \\
(7.82)\end{array}$ & $\begin{array}{l}65.77 \\
(8.14)\end{array}$ & $\begin{array}{l}56.97 \\
(7.53)\end{array}$ & $\begin{array}{l}46.67 \\
(6.87)\end{array}$ & $\begin{array}{l}57.33 \\
(7.60)\end{array}$ \\
\hline $\mathrm{T}_{5}$ & bakain & $\begin{array}{r}71.66 \\
(8.49)\end{array}$ & $\begin{array}{l}51.69 \\
(7.22)\end{array}$ & $\begin{array}{l}56.61 \\
(7.56) \\
\end{array}$ & $\begin{array}{l}47.01 \\
(6.89)\end{array}$ & $\begin{array}{l}37.51 \\
(6.17)\end{array}$ & $\begin{array}{l}48.20 \\
(6.98)\end{array}$ \\
\hline $\mathrm{T}_{6}$ & madar & $\begin{array}{l}69.33 \\
(8.36)\end{array}$ & $\begin{array}{l}44.75 \\
(6.73)\end{array}$ & $\begin{array}{l}49.75 \\
(7.09)\end{array}$ & $\begin{array}{l}40.15 \\
(6.38)\end{array}$ & $\begin{array}{l}30.65 \\
(5.58)\end{array}$ & $\begin{array}{l}43.04 \\
(6.60)\end{array}$ \\
\hline $\mathrm{T}_{7}$ & Lantana & $\begin{array}{l}75.66 \\
(8.73)\end{array}$ & $\begin{array}{l}49.70 \\
(7.09)\end{array}$ & $\begin{array}{l}54.70 \\
(7.43)\end{array}$ & $\begin{array}{l}45.10 \\
(6.75)\end{array}$ & $\begin{array}{l}35.60 \\
(6.00)\end{array}$ & $\begin{array}{l}46.27 \\
(6.84)\end{array}$ \\
\hline $\mathrm{T}_{8}$ & Datura & $\begin{array}{l}67.00 \\
(8.22)\end{array}$ & $\begin{array}{l}63.68 \\
(8.01)\end{array}$ & $\begin{array}{l}68.71 \\
(8.32) \\
\end{array}$ & $\begin{array}{l}59.11 \\
(7.72)\end{array}$ & $\begin{array}{l}49.61 \\
(7.08)\end{array}$ & $\begin{array}{l}60.27 \\
(7.80) \\
\end{array}$ \\
\hline $\mathrm{T}_{9}$ & Drumstick & $\begin{array}{l}70.33 \\
(8.42)\end{array}$ & $\begin{array}{l}55.05 \\
(7.45)\end{array}$ & $\begin{array}{l}60.23 \\
(7.79)\end{array}$ & $\begin{array}{l}50.63 \\
(7.15)\end{array}$ & $\begin{array}{l}41.13 \\
(6.45)\end{array}$ & $\begin{array}{l}51.76 \\
(7.23)\end{array}$ \\
\hline $\mathrm{T}_{10}$ & Congress grass & $\begin{array}{l}68.66 \\
(8.32)\end{array}$ & $\begin{array}{l}53.72 \\
(7.36)\end{array}$ & $\begin{array}{l}58.68 \\
(7.69)\end{array}$ & $\begin{array}{l}49.08 \\
(7.04)\end{array}$ & $\begin{array}{l}39.58 \\
(6.33)\end{array}$ & $\begin{array}{l}50.26 \\
(7.12)\end{array}$ \\
\hline $\mathrm{T}_{11}$ & Spinosad 45\% SC & $\begin{array}{r}71.33 \\
(8.48)\end{array}$ & $\begin{array}{l}56.40 \\
(7.54)\end{array}$ & $\begin{array}{l}61.37 \\
(7.87)\end{array}$ & $\begin{array}{l}51.77 \\
(7.23)\end{array}$ & $\begin{array}{l}42.27 \\
(6.54)\end{array}$ & $\begin{array}{l}52.95 \\
(7.31)\end{array}$ \\
\hline $\mathrm{T}_{12}$ & Control & $\begin{array}{l}74.50 \\
(8.61)\end{array}$ & $\begin{array}{l}85.00 \\
(9.93)\end{array}$ & $\begin{array}{r}100.00 \\
(9.99)\end{array}$ & $\begin{array}{l}116.50 \\
(10.89)\end{array}$ & $\begin{array}{c}105.66 \\
(10.30)\end{array}$ & $\begin{array}{r}101.79 \\
(9.60)\end{array}$ \\
\hline & S.Em \pm & 0.1675 & 0.1183 & 0.2187 & 0.2291 & 0.2763 & 0.2665 \\
\hline & C.D. $(5 \%)$ & N.S. & 0.3379 & 0.6364 & 0.6547 & 0.8100 & 0.7821 \\
\hline
\end{tabular}

NB: *figures in parentheses are square root $(\sqrt{x+0.5})$ transformed values. 
Table.3 Efficacy of phyto-pesticides against thrips infestation on tomato during rabi ,2018-19

\begin{tabular}{|c|c|c|c|c|c|c|c|}
\hline \multirow{3}{*}{$\begin{array}{l}\text { Treat. } \\
\text { Symbol }\end{array}$} & \multirow[t]{3}{*}{ Treatments } & \multicolumn{5}{|c|}{ Thrips/plant } & \multirow{3}{*}{$\begin{array}{l}\text { Overall mean } \\
\text { of two sprays }\end{array}$} \\
\hline & & & \multicolumn{2}{|c|}{$1^{\text {st }}$ spray } & \multicolumn{2}{|c|}{$2^{\text {nd }}$ spray } & \\
\hline & & 1 DBS & 7 DAS & 14 DAS & 7 DAS & 14 DAS & \\
\hline $\mathrm{T}_{1}$ & Castor & $\begin{array}{c}42.12 \\
6.49\end{array}$ & $\begin{array}{c}17.47 \\
4.18\end{array}$ & $\begin{array}{c}22.46 \\
4.74\end{array}$ & $\begin{array}{c}13.69 \\
3.76\end{array}$ & $\begin{array}{l}9.12 \\
3.02\end{array}$ & $\begin{array}{c}15.68 \\
4.02\end{array}$ \\
\hline $\mathrm{T}_{2}$ & Neem & $\begin{array}{c}43.42 \\
6.59\end{array}$ & $\begin{array}{c}13.76 \\
3.71\end{array}$ & $\begin{array}{c}18.74 \\
4.33\end{array}$ & $\begin{array}{c}10.24 \\
3.23\end{array}$ & $\begin{array}{l}5.47 \\
2.34\end{array}$ & $\begin{array}{c}12.05 \\
3.54\end{array}$ \\
\hline $\mathrm{T}_{3}$ & safeda & $\begin{array}{c}44.75 \\
6.69\end{array}$ & $\begin{array}{c}32.14 \\
5.67\end{array}$ & $\begin{array}{c}35.08 \\
6.09\end{array}$ & $\begin{array}{c}28.09 \\
5.36\end{array}$ & $\begin{array}{c}23.81 \\
4.88\end{array}$ & $\begin{array}{c}30.28 \\
5.54\end{array}$ \\
\hline $\mathrm{T}_{4}$ & Kaner & $\begin{array}{c}41.73 \\
6.46\end{array}$ & $\begin{array}{c}28.40 \\
5.33\end{array}$ & $\begin{array}{c}33.40 \\
5.78\end{array}$ & $\begin{array}{c}25.10 \\
5.01\end{array}$ & $\begin{array}{c}20.07 \\
4.48\end{array}$ & $\begin{array}{c}26.74 \\
5.21\end{array}$ \\
\hline $\mathrm{T}_{5}$ & bakain & $\begin{array}{c}43.03 \\
6.56\end{array}$ & $\begin{array}{c}22.09 \\
4.70\end{array}$ & $\begin{array}{c}27.04 \\
5.20\end{array}$ & $\begin{array}{c}18.74 \\
4.33\end{array}$ & $\begin{array}{c}13.76 \\
3.71\end{array}$ & $\begin{array}{c}20.40 \\
4.57\end{array}$ \\
\hline $\mathrm{T}_{6}$ & madar & $\begin{array}{c}44.08 \\
6.64\end{array}$ & $\begin{array}{c}15.44 \\
3.93\end{array}$ & $\begin{array}{c}20.43 \\
4.52\end{array}$ & $\begin{array}{c}12.11 \\
3.48\end{array}$ & $\begin{array}{l}7.12 \\
2.67\end{array}$ & $\begin{array}{c}13.77 \\
3.77\end{array}$ \\
\hline $\mathrm{T}_{7}$ & Lantana & $\begin{array}{c}44.75 \\
6.69\end{array}$ & $\begin{array}{c}19.71 \\
4.44\end{array}$ & $\begin{array}{c}24.80 \\
4.98\end{array}$ & $\begin{array}{c}16.48 \\
4.06\end{array}$ & $\begin{array}{c}12.81 \\
3.58\end{array}$ & $\begin{array}{c}18.45 \\
4.35\end{array}$ \\
\hline $\mathrm{T}_{8}$ & Datura & $\begin{array}{c}41.47 \\
6.44\end{array}$ & $\begin{array}{c}30.47 \\
5.52\end{array}$ & $\begin{array}{c}35.40 \\
5.95\end{array}$ & $\begin{array}{c}27.14 \\
5.21\end{array}$ & $\begin{array}{c}22.09 \\
4.70\end{array}$ & $\begin{array}{c}28.77 \\
5.41\end{array}$ \\
\hline $\mathrm{T}_{9}$ & Drumstick & $\begin{array}{c}42.77 \\
6.54\end{array}$ & $\begin{array}{c}24.40 \\
4.94\end{array}$ & $\begin{array}{c}29.48 \\
5.43\end{array}$ & $\begin{array}{c}21.16 \\
4.60\end{array}$ & $\begin{array}{c}16.08 \\
4.01\end{array}$ & $\begin{array}{c}22.78 \\
4.82\end{array}$ \\
\hline $\mathrm{T}_{10}$ & Congress grass & $\begin{array}{c}43.56 \\
6.60\end{array}$ & $\begin{array}{c}22.46 \\
4.74\end{array}$ & $\begin{array}{c}27.45 \\
5.24\end{array}$ & $\begin{array}{c}19.09 \\
4.37\end{array}$ & $\begin{array}{c}14.13 \\
3.76\end{array}$ & $\begin{array}{c}20.78 \\
4.61\end{array}$ \\
\hline $\mathrm{T}_{11}$ & Spinosad $45 \%$ SC & $\begin{array}{c}43.42 \\
6.59\end{array}$ & $\begin{array}{c}27.14 \\
5.21\end{array}$ & $\begin{array}{c}32.14 \\
5.67\end{array}$ & $\begin{array}{c}23.81 \\
4.88\end{array}$ & $\begin{array}{c}18.74 \\
4.33\end{array}$ & $\begin{array}{c}25.45 \\
5.06\end{array}$ \\
\hline $\mathrm{T}_{12}$ & Control & $\begin{array}{c}42.38 \\
6.51\end{array}$ & $\begin{array}{c}45.69 \\
6.76\end{array}$ & $\begin{array}{c}47.74 \\
6.91\end{array}$ & $\begin{array}{c}63.12 \\
7.90\end{array}$ & $\begin{array}{c}53.42 \\
7.34\end{array}$ & $\begin{array}{c}52.49 \\
7.24\end{array}$ \\
\hline & S.Em \pm & N.S. & 0.9017 & 1.1711 & 1.2519 & 1.2169 & 1.1954 \\
\hline & C.D. at $5 \%$ & 0.4041 & 0.3071 & 0.3992 & 0.3246 & 0.3669 & 0.2881 \\
\hline
\end{tabular}

$\mathrm{NB}: *$ figures in parentheses are square $\operatorname{root}(\sqrt{x+0.5})$ transformed values. 
Table.4 Efficacy of phyto-pesticides against fruit borer infestation on tomato during rabi ,2018-19

\begin{tabular}{|c|c|c|c|c|c|c|c|}
\hline \multirow{3}{*}{$\begin{array}{l}\text { Treat. } \\
\text { symbol }\end{array}$} & \multirow[t]{3}{*}{ Treatments } & \multirow[t]{3}{*}{1 DBS } & \multicolumn{4}{|c|}{ Fruit borer larval population per meter row } & \multirow{3}{*}{$\begin{array}{l}\text { Overall mean } \\
\text { of two sprays }\end{array}$} \\
\hline & & & \multicolumn{2}{|c|}{$1^{\text {st }}$ spray } & \multicolumn{2}{|c|}{$2^{\text {nd }}$ spray } & \\
\hline & & & 7 DAS & 14 DAS & 7 DAS & 14 DAS & \\
\hline $\mathrm{T}_{1}$ & Castor & $\begin{array}{c}3.72 \\
(2.05)\end{array}$ & $\begin{array}{c}3.07 \\
(1.89)\end{array}$ & $\begin{array}{c}3.77 \\
(2.07)\end{array}$ & $\begin{array}{c}2.87 \\
(1.84)\end{array}$ & $\begin{array}{c}2.27 \\
(1.66)\end{array}$ & $\begin{array}{c}2.99 \\
(1.87)\end{array}$ \\
\hline $\mathrm{T}_{2}$ & Neem & $\begin{array}{c}3.86 \\
(2.09)\end{array}$ & $\begin{array}{c}2.51 \\
(1.73)\end{array}$ & $\begin{array}{c}3.21 \\
(1.93)\end{array}$ & $\begin{array}{c}2.31 \\
(1.68)\end{array}$ & $\begin{array}{c}1.71 \\
(1.49)\end{array}$ & $\begin{array}{c}2.43 \\
(1.71)\end{array}$ \\
\hline $\mathrm{T}_{3}$ & Safeda & $\begin{array}{c}4.21 \\
(2.17)\end{array}$ & $\begin{array}{c}4.18 \\
(2.16)\end{array}$ & $\begin{array}{l}4.88 \\
(2.32)\end{array}$ & $\begin{array}{c}3.98 \\
(2.12)\end{array}$ & $\begin{array}{l}3.38 \\
(1.97)\end{array}$ & $\begin{array}{c}4.10 \\
(2.14)\end{array}$ \\
\hline $\mathrm{T}_{4}$ & Kaner & $\begin{array}{c}4.18 \\
(2.16)\end{array}$ & $\begin{array}{c}3.98 \\
(2.12)\end{array}$ & $\begin{array}{c}4.68 \\
(2.28)\end{array}$ & $\begin{array}{c}3.78 \\
(2.07)\end{array}$ & $\begin{array}{c}3.18 \\
(1.92)\end{array}$ & $\begin{array}{c}3.90 \\
(2.10)\end{array}$ \\
\hline $\mathrm{T}_{5}$ & bakain & $\begin{array}{c}3.97 \\
(2.11)\end{array}$ & $\begin{array}{c}3.63 \\
(2.03)\end{array}$ & $\begin{array}{c}4.33 \\
(2.20)\end{array}$ & $\begin{array}{c}3.43 \\
(1.98)\end{array}$ & $\begin{array}{c}2.83 \\
(1.82)\end{array}$ & $\begin{array}{c}3.55 \\
(2.01)\end{array}$ \\
\hline $\mathrm{T}_{6}$ & madar & $\begin{array}{c}3.68 \\
(2.04)\end{array}$ & $\begin{array}{c}2.81 \\
(1.82)\end{array}$ & $\begin{array}{c}3.51 \\
(2.00)\end{array}$ & $\begin{array}{c}2.61 \\
(1.76)\end{array}$ & $\begin{array}{l}2.01 \\
(1.58)\end{array}$ & $\begin{array}{c}2.73 \\
(2.03)\end{array}$ \\
\hline $\mathrm{T}_{7}$ & Lantana & $\begin{array}{c}4.20 \\
(2.17)\end{array}$ & $\begin{array}{c}3.58 \\
(2.02)\end{array}$ & $\begin{array}{c}4.28 \\
(2.19)\end{array}$ & $\begin{array}{c}3.38 \\
(1.94)\end{array}$ & $\begin{array}{c}2.78 \\
(1.81)\end{array}$ & $\begin{array}{c}3.61 \\
(2.13)\end{array}$ \\
\hline $\mathrm{T}_{8}$ & Datura & $\begin{array}{c}4.31 \\
(2.19)\end{array}$ & $\begin{array}{c}4.01 \\
(2.12)\end{array}$ & $\begin{array}{c}4.71 \\
(2.28)\end{array}$ & $\begin{array}{c}3.81 \\
(2.08)\end{array}$ & $\begin{array}{c}3.21 \\
(1.93)\end{array}$ & $\begin{array}{c}4.05 \\
(2.06)\end{array}$ \\
\hline $\mathrm{T}_{9}$ & Drumstick & $\begin{array}{c}4.12 \\
(2.15)\end{array}$ & $\begin{array}{c}3.83 \\
(2.08)\end{array}$ & $\begin{array}{c}4.53 \\
(2.24)\end{array}$ & $\begin{array}{c}3.63 \\
(2.03)\end{array}$ & $\begin{array}{l}3.03 \\
(1.88)\end{array}$ & $\begin{array}{c}3.75 \\
(2.03)\end{array}$ \\
\hline $\mathrm{T}_{10}$ & Congress grass & $\begin{array}{c}3.93 \\
(2.10)\end{array}$ & $\begin{array}{c}3.71 \\
(2.05)\end{array}$ & $\begin{array}{c}4.41 \\
(2.22)\end{array}$ & $\begin{array}{c}3.51 \\
(2.00)\end{array}$ & $\begin{array}{c}2.91 \\
(1.85)\end{array}$ & $\begin{array}{c}3.63 \\
(1.300)\end{array}$ \\
\hline $\mathrm{T}_{11}$ & $\begin{array}{c}\text { Spinosad } 45 \% \\
\text { SC }\end{array}$ & $\begin{array}{c}4.08 \\
(2.14)\end{array}$ & $\begin{array}{c}1.27 \\
(1.33)\end{array}$ & $\begin{array}{c}1.97 \\
(1.57)\end{array}$ & $\begin{array}{c}1.07 \\
(1.25)\end{array}$ & $\begin{array}{c}0.47 \\
(0.98)\end{array}$ & $\begin{array}{c}1.19 \\
(2.45)\end{array}$ \\
\hline $\mathrm{T}_{12}$ & Control & $\begin{array}{c}3.92 \\
(2.10)\end{array}$ & $\begin{array}{c}4.12 \\
(2.15)\end{array}$ & $\begin{array}{c}5.27 \\
(2.40)\end{array}$ & $\begin{array}{c}7.54 \\
(2.58)\end{array}$ & $\begin{array}{c}6.76 \\
(2.69)\end{array}$ & $\begin{array}{c}5.92 \\
(2.92)\end{array}$ \\
\hline & S.Em \pm & 0.0548 & 0.0837 & 0.0876 & 0.1291 & 0.0730 & 0.1091 \\
\hline & C.D. at $5 \%$ & N.S. & 0.2445 & 0.2592 & 0.3795 & 0.2151 & 0.3187 \\
\hline
\end{tabular}

$\mathrm{NB}: *$ figures in parentheses are square $\operatorname{root}(\sqrt{x+0.5})$ transformed values. 
Int.J.Curr.Microbiol.App.Sci (2021) 10(05): 524-537

Fig.1 Bio-efficacy of phyto-pesticides on aphid after first spray

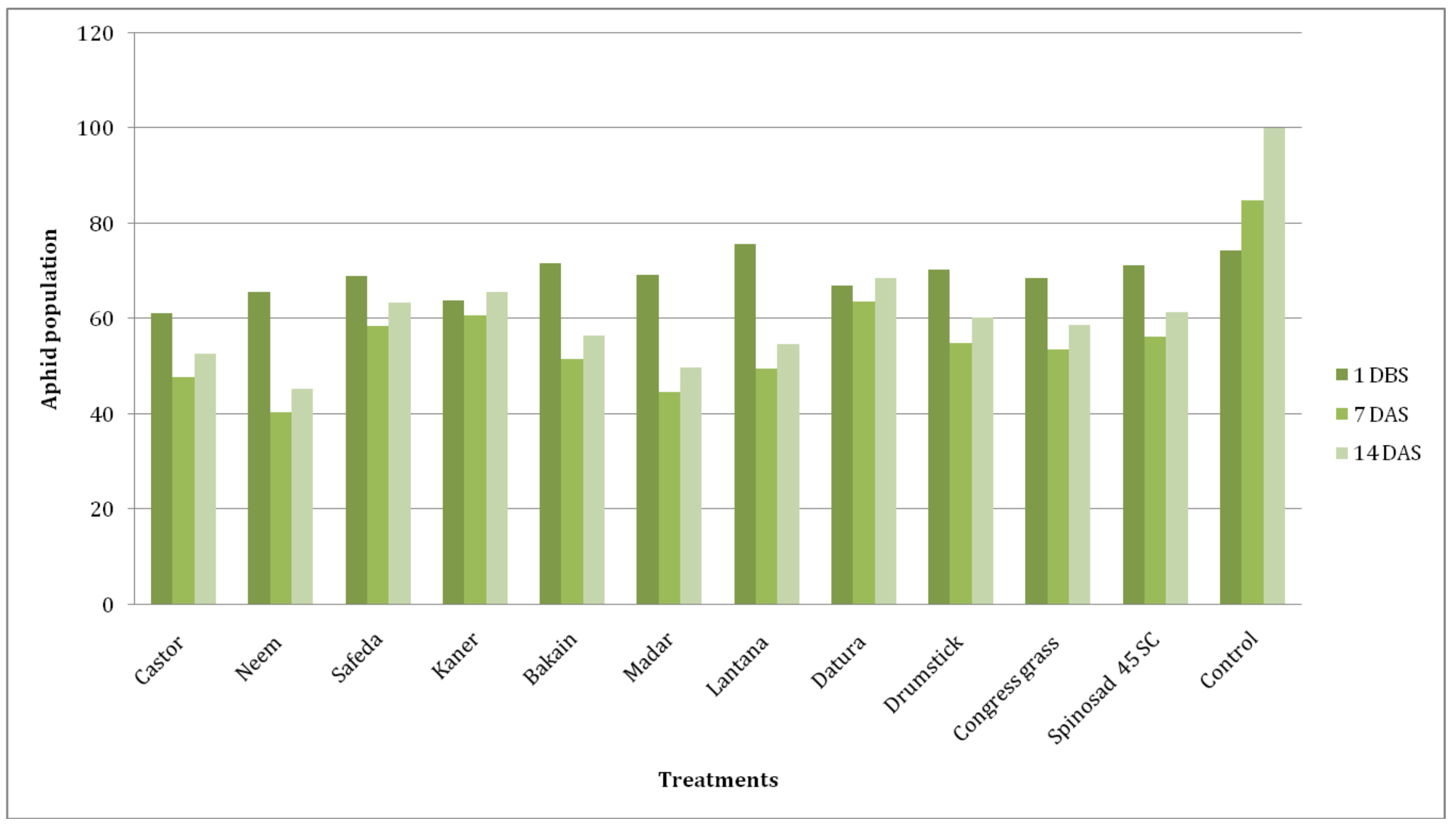


Int.J.Curr.Microbiol.App.Sci (2021) 10(05): 524-537

Fig.2 Bio-efficacy of phyto-pesticides on aphid after second spray

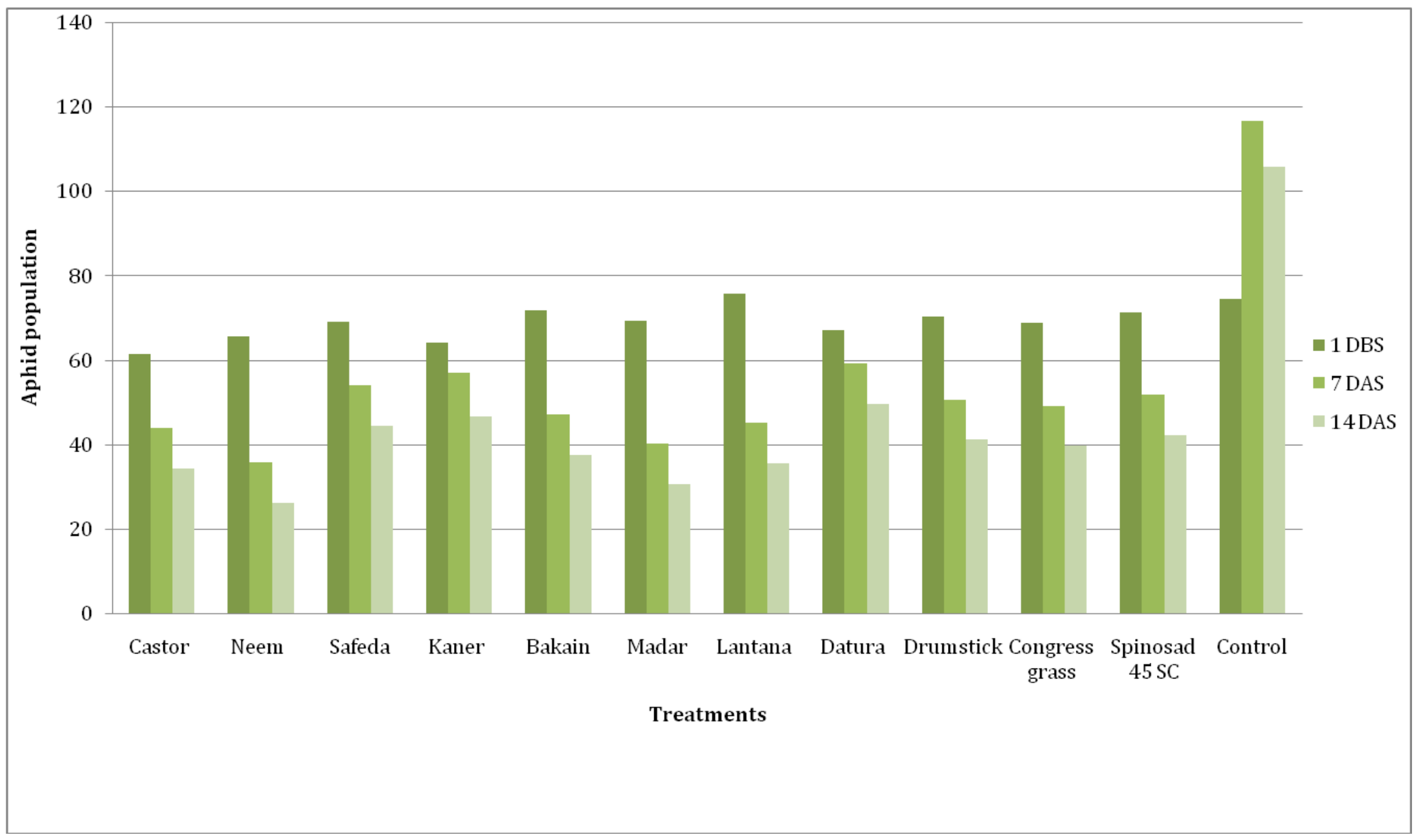


Fig.3 Bio-efficacy of phyto-pesticides on mean population of thrips after first spray

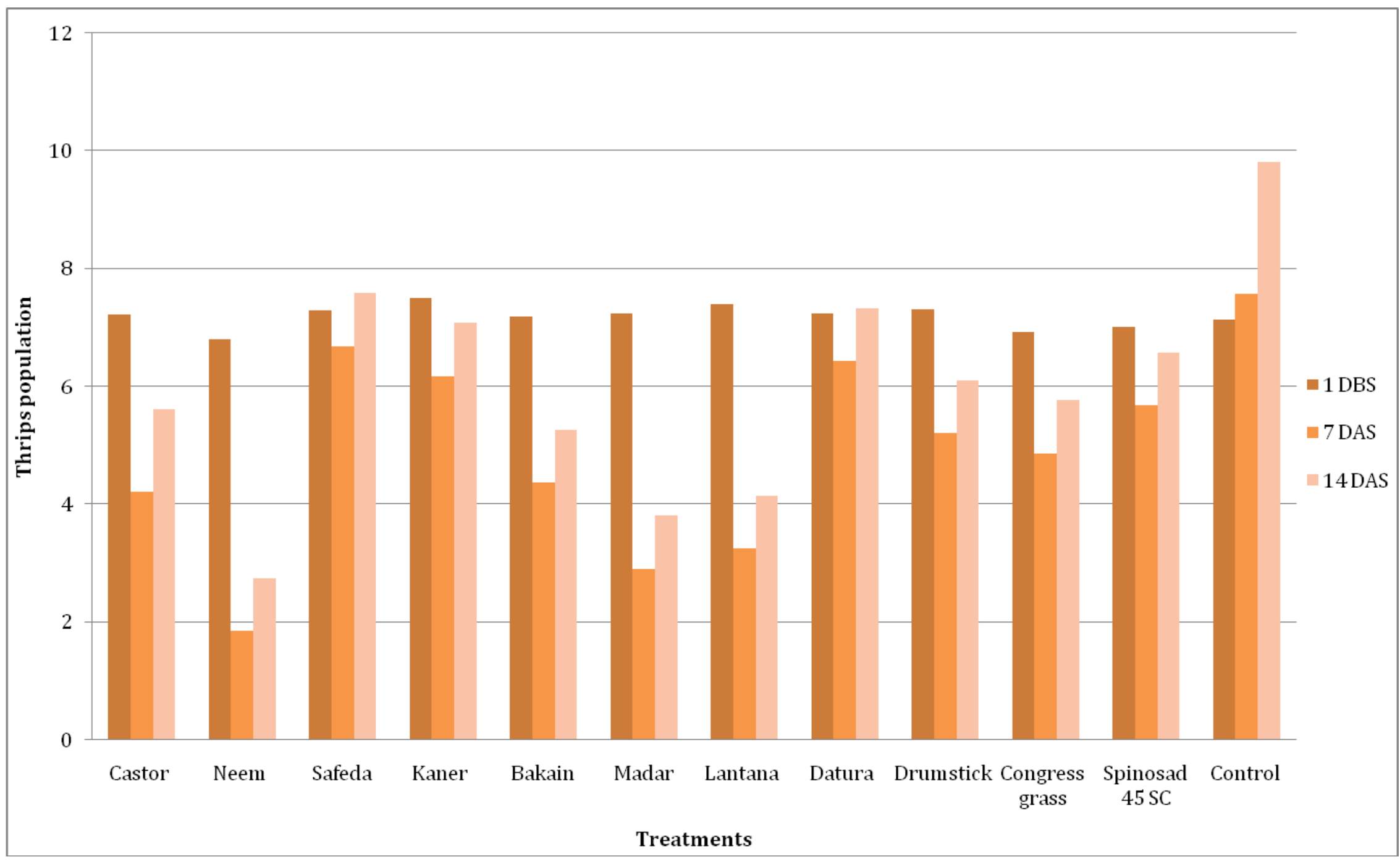


Fig.4 Bio-efficacy of phyto-pesticides on mean population of thrips after second spray

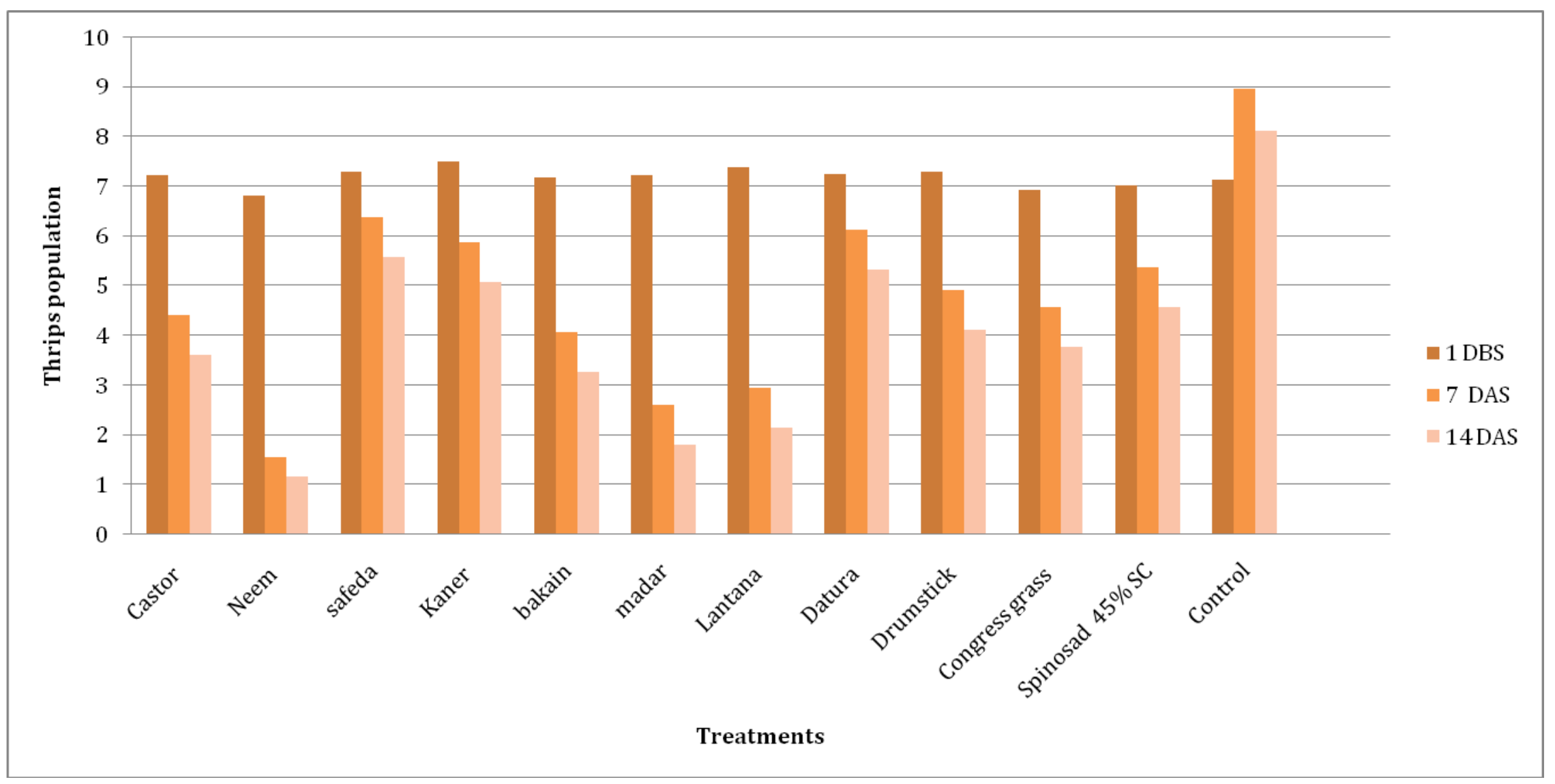


Fig.5 Bio-efficacy of phyto-pesticides on mean population of fruit borer after first spray

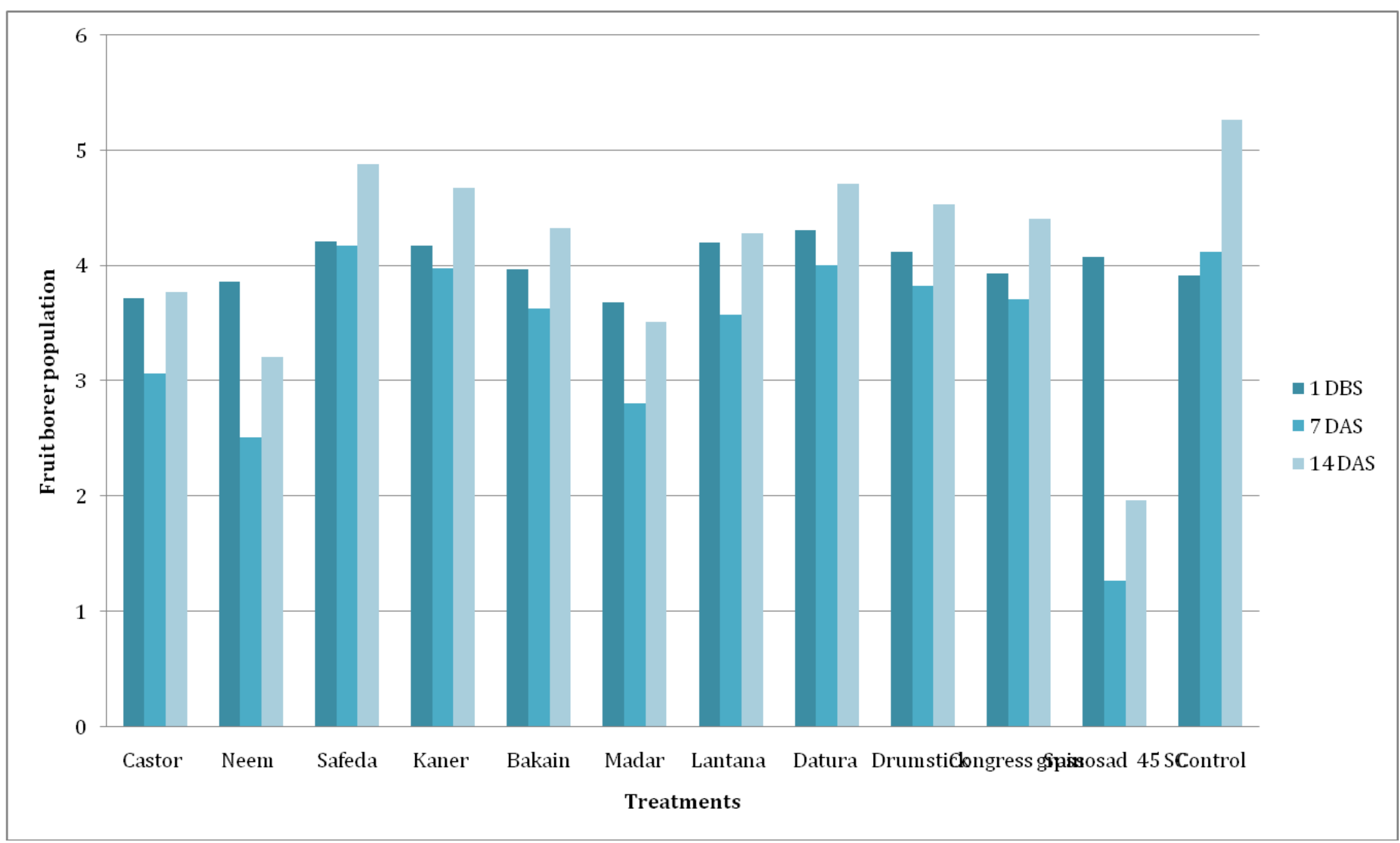


Fig.6 Bio-efficacy of phyto-pesticides on mean population of fruit borer after first spray

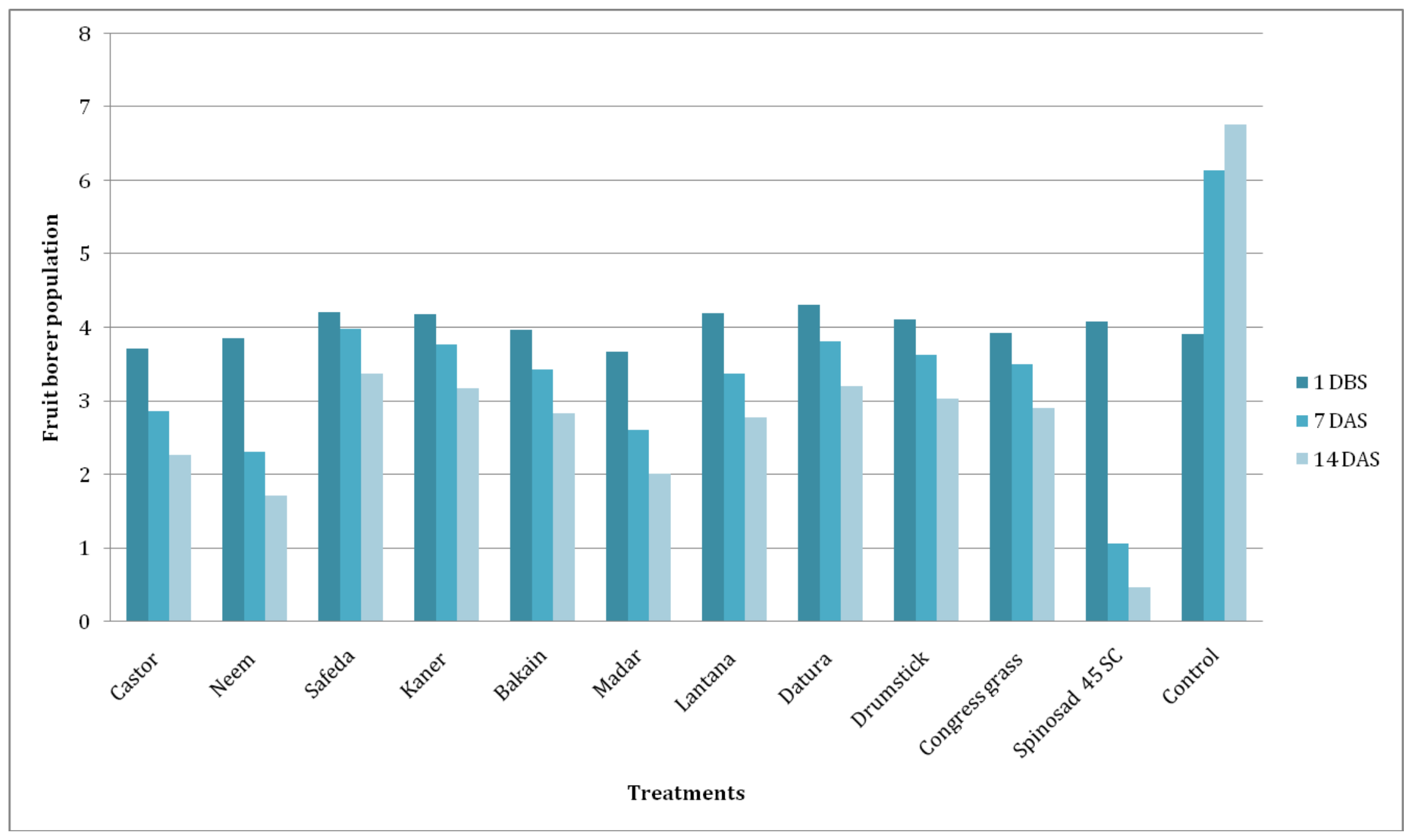


Ranking of different phyto-pesticides based on overall aphid intensity was found to be neem < madar < castor < lantana < bakain < congress grass < drumstick < safeda < kaner < datura. However application of spinosad 45 SC $(0.015 \%)$ was not found suitable for aphid management as all the phyto-pesticides received lower intensity of aphid except safeda and datura. These findings were in agreement with the findings of Nzanza and Mashela (2012), who reported the effects of fermented plant extracts of neem leaf and wild garlic (Tulbaghia violacea) show effective control of $B$. tabaci and A. gossypii population.

\section{Thrips (Scirtothrips dorsalis Hood)}

Data depicted in table 3 on thrips intensity revealed that the overall mean population was calculated considering the average of all observations which varied between 12.05 to 52.49 thrips/plant. Neem leaf decoction treated tomato crop received significantly lowest thrips intensity 12.05 thrips/plant which was on par with 13.77, 15.68 and 18.45 thrips/plant recorded on madar, castor and lantana leaves decoction sprayed crop respectively. Ranking of different phytopesticides based on overall thrips intensity was found to be neem < madar < castor < lantana $<$ bakain < congress grass < drumstick < safeda $<$ kaner < datura. However application of spinosad $45 \mathrm{SC}(0.015 \%)$ was not found suitable for thrips management as all the phyto-pesticides received lower intensity of thrips except safeda and datura.

\section{Fruit borer (Helicoverpa armigera Hubner)}

Data depicted in table 4 on fruit borer intensity revealed that the overall mean population of fruit borer was calculated considering the average of all observations, which varied between 1.19 to 5.92 fruit borer larvae/m row length. Neem leaf decoction treated tomato crop received significantly lowest fruit borer intensity being 1.19 fruit borer larvae/m row length, which was on par with $2.43,2.73$ and 2.99 fruit borer larvae/m row length recorded on neem, madar and castor leaves concoction sprayed crop, respectively.

Ranking of different phyto-pesticides based on overall fruit borer intensity was found to be neem < madar < castor < bakain < lantana < congress grass $<$ drumstick $<$ kaner $<$ datura $<$ safeda. However, application of spinosad 45 SC $(0.015 \%)$ was found best suitable for fruit borer management among the treatments. Work done by earlier researchers is in conformity with the present findings. Mahadevan (1998) revealed that spraying 2 rounds of neem products was the best means of controlling the pest.

Jayakumar and Gupta (1999) reported that NSKE reduced the oviposition of $H$. armigera in a dependant manner during the exposure period of 0.24 hours and 24-28 hours and showed oviposition deterrence effect. Reduction in oviposition was highest $(60.9 \%)$ with (10 \%) NSKE. Vyas et al., (1999) found that extracts of neem (A. indica, A. Juss) had a significant effect on $H$. armigera.

It can be concluded that the tomato crop treated with neem leaf concoction@5\% had significantly lowest aphid and thrips population followed by madar, castor and lantana treated crop against the highest average aphid and thrips intensity of 101.79 and 52.49/plant on untreated crop, respectively. Spinosad 45 SC $(0.015 \%)$ treated tomato crop had significantly lowest fruit borer intensity followed by neem, madar, castor treated crop against the highest average fruit borer intensity 5.92/ $\mathrm{m}$ row length on untreated crop.

\section{References}

Abou-Yousef, H. M.; Farghaly, F. S., and Torkey H. M., 2010. Insecticidal activity of 
some plant extracts against some sapsucking insects under laboratory conditions. World Journal Agriculture Science. 6 (4): 434- 439.

Dhandapani N, Umeshchandra S R, Murugan M., 2003. Bio-intensive pest management (BIPM) in major vegetable crops: $A n$ Indian perspective. Food Agriculture Environment. 1: 333-339

Gore N. and Sreenivasa M. N. 2011. Influence of liquid organic manures on growth, nutrient content and yield of tomato (Lycopersicon esculentum) in the sterilized soil. Karnataka journal agriculture science. 24(2): 153-157.

Gurjar M., Ali S., Akhtar M. and Singh S. 2012. Efficacy of plant extracts in plant disease management. 3(3): 425-433.

Hassan S. A. 1992. Guidelines for testing the effects of pesticides on beneficial organisms: Description of test methods. The International organization for biological control : WPRS, Bulletin. 15(3): 18-39

Jayakumar, P. and Gupta, G. P., 1999. Effect of neem seed kernal extract (NSKE) on Helicoverpa armigera. Pesticide Research Journal 11(1): 32-36.

Kumar, N. K. K. 1995. Yield loss in chilli and sweet pepper due to Scirtothrips dorsalis Hood (Thysanoptera: Thripidae). Pest Management in Horticultural Ecosystems. 1: 61-69.

Mahadevan, N. R. 1998. Azadirachtin to control tomato borer. Insect Environment. 3 (4): 9.

Marasini P. and Paudel S. 2017. Phenotypic characterization of tomato (Lycopersicon esculentum) Journal horticulture. 4(3): 1-4.

Meena L K and Raju S V S 2014. Bio-efficacy of newer insecticides against tomato fruit borer, Helicoverpa armigera (Hubner) on tomato, Lycopersicon esculentum Mill under field conditions. The Bioscan 9(1): 347-350.

Mehta R. 2017. History of tomato (Poor man`s apple). Journal of humanities and animal science. 22(8): 31-34

Nzanza, B. and Mashela, P. W. 2012. Control of whiteflies and aphids in tomato (Solanum lycopersicum L.) by fermented plant extracts of neem leaf and wild garlic. African Journal of Biotechnology. 11 (94): 16077-16082.

Prabhu, M. J. 2009.Panchagavya: low cost organic input for both crops and animals in Farmer's Notebook, The Hindu Online Edition, 4th June, 2009.

Reddy, N. A and Kumar, C. T. A 2004. Insect pests of tomato, Lycopersicon esculentum Mill. in eastern dry zone of Karnataka. Insect Environment. 10 (1): 40-42.

Sangeetha V, Thevanathan R. 2010. Effect of Panchagavya on Nitrate Assimilation by Experimental Plants. Journal American Science.6 (2):76-82.

Shah, J. A.; Mian Inayatullah; Kamran Sohail; Shah, S. F.; Suleman Shah; Toheed Iqbal and Muhammad U. 2013. Efficacy of botanical extracts and a chemical pesticide against tomato fruit worm, Helicoverpa armigera. Sarhad Journal of Agriculture. 29 (1): 93-96.

Sutton, A. 1991. Tomato vegetable market development team, CIBA-GEIGY. AG 6(82) Basel. p. 660.

Vyas, B. N.; Ganesan, S., Raman, K.; Godrej, N. B.; Mistry, K. B.; Singh P. P.; and Saxena R. C. 1999. Effect of three plant extracts on growth and development of three noctuid pests. Azadirachta indica A. Juss. 17(5):103-109.

\section{How to cite this article:}

Ajay Deep Singh, P. Prathyusha, Y. P. Malik and Dwivedi, R. K. 2021. Bio-Efficacy of PhytoPesticides against Major Insect- Pest of Organically Grown Tomato. Int.J.Curr.Microbiol.App.Sci. 10(05): 524-537. doi: https://doi.org/10.20546/ijcmas.2021.1005.060 